\title{
On the shape of luminosity profiles of dwarf galaxies as a distance indicator: The NGC 5044 Group revisited ${ }^{\star}$
}

\author{
S. A. Cellone ${ }^{1}$ and A. Buzzoni ${ }^{2,3}$ \\ 1 Facultad de Ciencias Astronómicas y Geofísicas, Universidad Nacional de La Plata, Paseo del Bosque, \\ 1900 La Plata, Argentina \\ 2 Telescopio Nazionale Galileo, Roque de los Muchachos Astronomical Obs., A.P. 565, \\ 38700 S/Cruz de La Palma (TF), Spain \\ e-mail: buzzoni@tng.iac.es \\ 3 Osservatorio Astronomico di Brera, Via Bianchi 46, 23807 Merate (Lc), Italy
}

Received 15 February 2000 / Accepted 4 December 2000

\begin{abstract}
Conflicting evidence has been recently raised in order to use surface brightness profiles of dwarf galaxies as a distance indicator. In this paper we discuss in some detail the main error sources in profile fitting procedures for galaxies with more than one physical component, showing their impact on the resulting shape parameters. The apparent tight coupling between shape parameter and (pseudo) scalelength in the Sérsic law is especially dealt with, demonstrating that this relationship is mostly a mathematical artifact, thus throwing doubts on its usefulness as a distance indicator. Galaxies departing from the luminosity-shape relation are shown to exhibit different kinds of intrinsic peculiarities, thus prompting for better securing the empirical constraints to conform observational samples. As a relevant example in this sense, new observations of the galaxy N50 in the NGC 5044 Group are presented. We show that this object may be at an intermediate evolutionary stage between blue compact dwarfs (BCDs) and dwarf ellipticals (dEs).
\end{abstract}

Key words. galaxies: clusters: NGC 5044 Group - galaxies: distances and redshifts, elliptical and lenticular, fundamental parameters, photometry, structure

\section{Introduction}

The shape of the surface brightness profiles of elliptical galaxies, quantified by the parameter $N$ in the Sérsic (1968) law

$I_{(r)}=I_{0} \mathrm{e}^{-(r / \alpha)^{N}}$,

has been shown to correlate with luminosity, both for dwarf (Young \& Currie 1994, hereafter YC94; Cellone et al. 1994; Durrell et al. 1996; Durrell 1997) and bright systems (Caon et al. 1993; D'Onofrio et al. 1994).

The use of this $L-N$ relationship as a distance indicator for dwarf elliptical galaxies was first investigated by YC94, while the correlation between the Sérsic parameters $N$ and $\alpha$ was subsequently used (Young \& Currie

Send offprint requests to: S. A. Cellone,

e-mail: scellone@f caglp.unlp.edu.ar

* Based on observations collected at the European Southern Observatory, La Silla (Chile), and the Complejo Astronómico El Leoncito (CASLEO), operated under agreement between CONICET and the Universities of La Plata, Córdoba, and San Juan (Argentina).
1995, YC95) to derive the distances to 64 dwarf galaxies in the Virgo Cluster. The quest about the real usefulness of the $L-N$ and $\alpha-N$ relations to determine extragalactic distances has received opposite arguments in favour (Young \& Currie 1998) or against (Binggeli \& Jerjen 1998, BJ98).

In a recent paper, Cellone $(1999$, C99) presented CCD surface photometry for a small sample of dwarf and intermediate luminosity galaxies in the NGC 5044 Group, showing that, at least in that case, the $L-N$ correlation partially fails due to a few relatively bright galaxies with "convex" profiles (i.e. $N>1$ ) strongly deviating from the standard relationship. In addition, the important intrinsic scatter in the $L$ vs. $N$ trend seems to drastically limit its practical use for obtaining the distance to the group.

A re-elaboration of a subset of those data, led Young \& Currie (2001, YC00) to conclude that the galaxy population in the NGC 5044 Group displays on the contrary "a tight scalelength-shape relationship" revealing therefore to be "an excellent distance indicator".

As we will show in the present paper, there are reasons to believe that most of the $\mathrm{YC} 00$ conclusions rest 
in fact on a misinterpretation of the data (Sect. 2), while their claimed apparent tightness of the $\alpha-N$ relation seems likely an artifact of the parameter mathematical coupling in Eq. (1). This would therefore throw serious doubts in using this method to derive extragalactic distances (Sect. 4.1).

We will also turn back here to the relevant case of galaxy N50 in the NGC 5044 Group, one of those "outliers" escaping the standard $L-N$ relation in Cellone's (1999) analysis. In spite of its ostensibly normal dE photometric properties, new accurate observations (Sect. 3) indicate indeed that this is a quite peculiar and interesting object sharing most of the characters of dwarf ellipticals $(\mathrm{dE})$ and blue compact dwarf (BCD) galaxies.

\section{The art of profile fitting}

Suitable fitting of radial profiles in low surface brightness galaxies is a delicate task which, like an art, requires good skills and the knowledge of the appropriate technique. Every error sources, from seeing and photon noise, as well as any uncertainty in the sky cleaning must be accounted for, and we have to pay attention at the same time also to preserve astrophysical self-consistency of our output.

In a fit of digitized plots of the NGC 5044 galaxy sample from Fig. 1 in C99, Young \& Currie (2001) obtained, for each galaxy, a new set of Sérsic parameters. Like C99, they worked in the surface brightness domain, that is by using Eq. (1) in the form

$S_{(r)}=S_{0}+1.086\left(\frac{r}{\alpha}\right)^{N}$

with $S_{(r)}$ in mag $\operatorname{arcsec}^{-2}$. In particular, they obtained very different $N$ (i.e., shape) values for galaxies $\mathrm{N} 42^{1}$, N51, and N95A, with respect to C99's results. According to their results, YC00 claim that "none of the three relatively bright galaxies cited as possessing convex profiles actually has a convex profile", meaning the three "outliers" N42, N49, and N50.

One of the questioned objects in the C99 sample is galaxy N42, for which C99 indicates $N=1.43$ to be compared with $N=0.60$ in the YC00 fit. At least two evident weak points emerge, in our opinion, from the $\mathrm{YC00}$ analysis. Contrary to the C99 fitting procedure, that only relied on the $S / N>1$ portion of galaxy surface brightness profile (i.e. with $I(r)>\sigma_{\mathrm{SKY}}$ ), the new fit extends much farther from galaxy centre. In the outermost regions, photon noise and statistical uncertainty in the sky subtraction begin to dominate causing the output profile to artificially level off at large radii. By itself, this effect works in inducing nominally "concave" (i.e., $N<1$ ) surface brightness profiles throughout in the YC00 fit (see Andredakis et al. 1995 for similar conclusions dealing with bulge deconvolution in spiral galaxies).

${ }^{1}$ Galaxy identification numbers throughout the paper are from the Ferguson \& Sandage (1990) catalogue of the NGC 5044 Group.
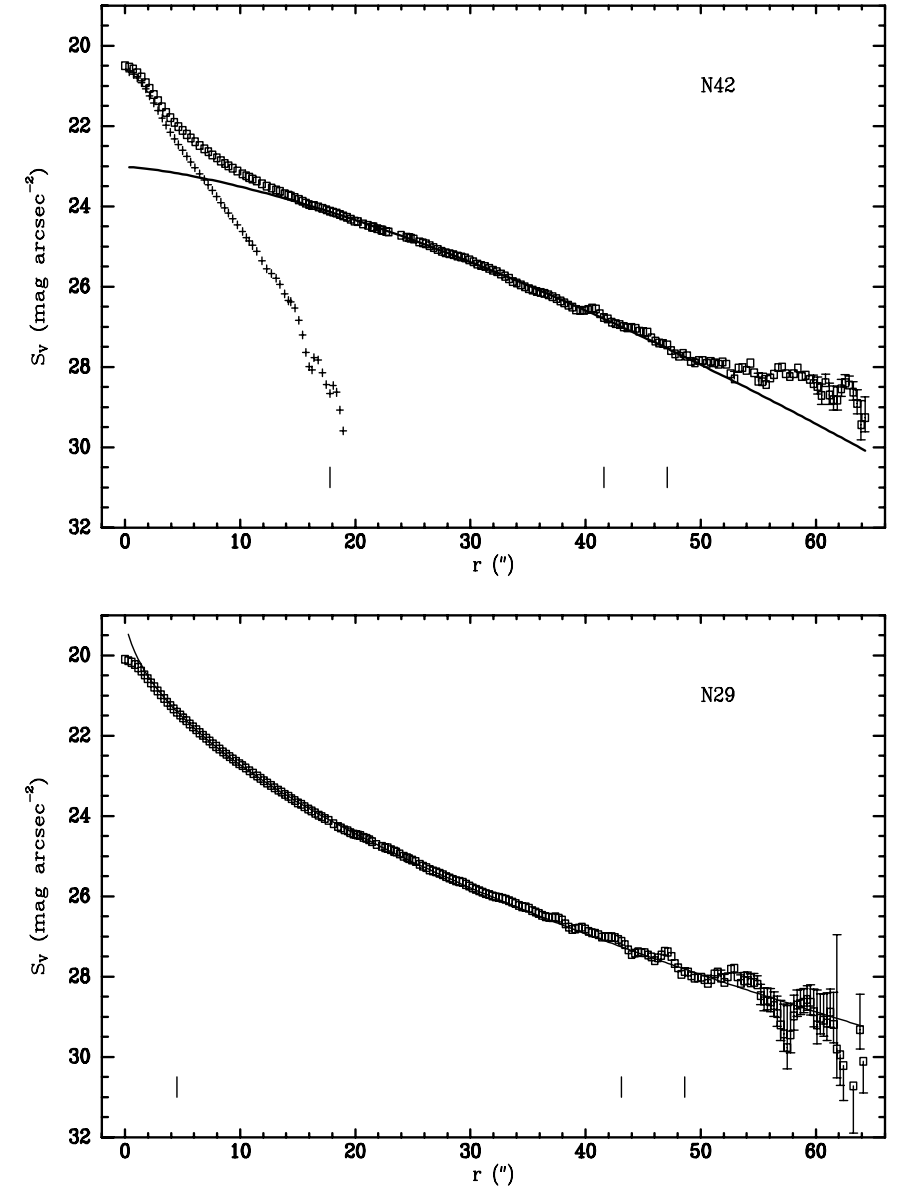

Fig. 1. $V$ band surface brightness profiles of galaxies N42 (top) and N29 (bottom). Sérsic law fits are shown as solid lines. Small ticks show the inner and the two outer $\left(1 \sigma_{\mathrm{SKY}}\right.$ and $\left.\sigma / 2_{\mathrm{SKY}}\right)$ radius cutoffs in C99 model fitting. Crosses show the bulge component of N42 after model subtraction. The fitting shape parameter $N$ results 1.43 for N42 and 0.54 for N29

In addition, even at first glance (cf. Fig. 1) the N42 surface brightness profile reveals at least two distinct components: an inner bulge and a main body extending out to $\sim 50^{\prime \prime}$. This galaxy would therefore need a multicomponent scheme (e.g.: Papaderos et al. 1996) to properly decompose its profile. Cellone's (1999) model for the N42 main component provided an integrated magnitude $V_{\mathrm{T}}=15.3$ mag. After subtraction, this leaves the inner bulge component, with $V_{\text {bulge }}=16.4$, and extending out to $r \simeq 17^{\prime \prime}$, as evident from Fig. 1 . Although this decomposition scheme might probably be not unique, it shows however that the main morphological component of the galaxy, providing about $3 / 4$ of the total $V$ luminosity, is in fact suitably fitted by the original "convex" profile. Our choice is also supported by a $\chi^{2}$ test on the fitting residuals confirming that a simple Sérsic fit can be ruled out at a $95 \%$ confidence level.

As a comparison, Fig. 1 (lower panel) also shows the profile of the bright dwarf N29. In this case, no change in slope is evident, and a single Sérsic law (with 
$N=0.54)$ fits nicely this profile all along its useful range, as confirmed again by the $\chi^{2}$ statistics.

The case of N51 (the second galaxy disputed by YC00) is similar to that of N42, although not so extreme, while for the third object, the previously uncatalogued galaxy N95A, C99 reported an exceedingly low surface brightness $\left(S_{0} \sim 24 \mathrm{mag} \operatorname{arcsec}^{-2}\right)$ that definitely prevented any reliable fit. For this reason this galaxy was not included in any subsequent analysis.

While statistical tests support in our case both the choice of a two-component fit for N42, and a simple Sérsic law for N29, more generally any suitable correction for the bulge contribution in dwarf galaxies may be a nonunivocal task. Seeing conditions and other internal bias sources (e.g. ongoing star formation) act in facts in the sense of disturbing galaxy morphology making any fitting procedure somewhat dependent on galaxy apparent size and on environment conditions as well.

In spite of any standard criterion to single out the bulge component, it is clear however that by simply neglecting the problem one would more likely tend to predict too "spiked" galaxy profiles preferring lower values of $N$ (Andredakis et al. 1995). We will turn back on this point and its impact on the $L-N$ relationship in Sect. 4 .

\section{N50: A link between BCDs and dEs?}

The galaxy N50 was originally classified as dEpec, N/BCDring by Ferguson \& Sandage (1990). Because of poor spatial resolution, C99 failed to detect any $\mathrm{BCD}$ feature in this object eventually appearing as a normal $\mathrm{dE}$, based also on its $(B-V)_{0}=0.76$ (cf. Caldwell \& Bothun 1987). In any case, this would make $\mathrm{N} 50$ an interesting object because it is, along with N42, one of the two brightest $\mathrm{dEs}$ with convex profile (i.e., $N>1$ ) in the C99 sample.

To better assess its evolutionary properties, we collected new observations of N50 with the EFOSC2 camera at the ESO $3.6 \mathrm{~m}$ telescope in La Silla, Chile, on the nights of April 16 and 17, 1999, as a part of a study of the low surface brightness galaxy population of the NGC 5044 Group. A detailed description of the observations and image processing will be given in a forthcoming paper (Buzzoni et al. 2001). Direct images of this galaxy were obtained under sub-arcsec seeing conditions in the $g, r, i$, and $z$ bands of the Gunn system (Schneider et al. 1983). Data reduction has been accomplished using the $I_{R A F}{ }^{2}$ package achieving a $\pm 0.001 \mathrm{mag}$ internal error, while external magnitude uncertainty from standard zero points amounted to $\pm 0.03 \mathrm{mag}$. Surface brightness profiles have been obtained in the four bands down $S_{\mathrm{g}} \sim 28 \mathrm{mag} \operatorname{arcsec}^{-2}$.

\footnotetext{
2 The Image Analysis and Reduction Facility is made available to the astronomical community by the National Optical Astronomy Observatories, operated by the Association of Universities for Research in Astronomy (AURA), Inc., under contract with the U. S. National Science Foundation.
}

Table 1. Gunn photometry of N50

\begin{tabular}{lcccc}
\hline \multicolumn{5}{c}{ Aperture magnitudes* } \\
radius [ $\left.{ }^{\prime \prime}\right]$ & $g$ & $g-r$ & $g-i$ & $g-z$ \\
\hline $2 .^{\prime \prime} 5$ & 17.554 & 0.436 & 0.697 & 0.610 \\
$5^{\prime \prime}$ 0 & 16.384 & 0.417 & 0.666 & 0.582 \\
$10^{\prime \prime}$ & 15.628 & 0.416 & 0.669 & 0.582 \\
$15^{\prime \prime}$ & 15.347 & 0.416 & 0.667 & 0.582 \\
$20^{\prime \prime}$ & 15.250 & 0.413 & 0.661 & 0.574 \\
$30^{\prime \prime}$ & 15.213 & 0.412 & 0.654 & 0.575 \\
\hline & \multicolumn{5}{c}{ Knot photometry* } \\
& $g$ & $g-r$ & $g-i$ & $g-z$ \\
\hline K-1 & 19.272 & 0.482 & 0.757 & 0.652 \\
K-2 & 19.786 & 0.360 & 0.572 & 0.517 \\
K-3 & 19.862 & 0.374 & 0.609 & 0.520 \\
K-4 & 19.779 & 0.381 & 0.614 & 0.554 \\
K-5 & 19.950 & 0.398 & 0.628 & 0.554 \\
K-6 & 19.797 & 0.416 & 0.646 & 0.590 \\
K-7 & 20.029 & 0.429 & 0.682 & 0.598 \\
K-8 & 19.820 & 0.474 & 0.755 & 0.662 \\
K-9 & 19.750 & 0.399 & 0.635 & 0.551 \\
K-10 & 19.913 & 0.409 & 0.647 & 0.564 \\
\hline
\end{tabular}

(*) Internal mag uncertainty is 0.001 for aperture photometry and 0.004 for knot magnitudes. Zero-point external error is 0.03 mag throughout.

Direct imaging has been complemented also with longslit spectroscopy between 4300 and $6300 \AA$ at $6 \AA$ FWHM wavelength resolution. Supplementary spectroscopic observations have been also carried out in the range 3500 $5400 \AA$ at $8 \AA$ FWHM resolution with a Boller \& Chivens spectrograph at the $2.15 \mathrm{~m}$ telescope of the CASLEO observatory in San Juan, Argentina on April 9, 1997.

Figure 2 (top panel) shows a $1^{\prime} \times 1^{\prime} g$ band contour plot of N50 (the stellar PSF FWHM is $0^{\prime \prime} 96$ ). The central $r \leq 3^{\prime \prime} .5\left(400 h_{0}^{-1} \mathrm{pc}\right)$ region shows several knots surrounding a central cusp. These features are better seen after subtraction of a Sérsic model (bottom left panel), and even a probable dust lane can be appreciated west of the nucleus. An enlarged map that identifies the central knots is also reported in the figure (bottom right).

Table 1 reports a full summary of the galaxy photometry, including aperture magnitudes and detailed measurements of the single knots. For the latter features, we tried different clean-up procedures to subtract the smooth galaxy contribution; however, a plain subtraction of the local "background" measured around each source eventually revealed the best choice. The internal photometric error amounted in this case to \pm 0.004 mag in each band.

The $g$ surface brightness profile of N50 is shown in Fig. 3. Error bars including photon noise and sky level uncertainty have been taken into account in the plot. 


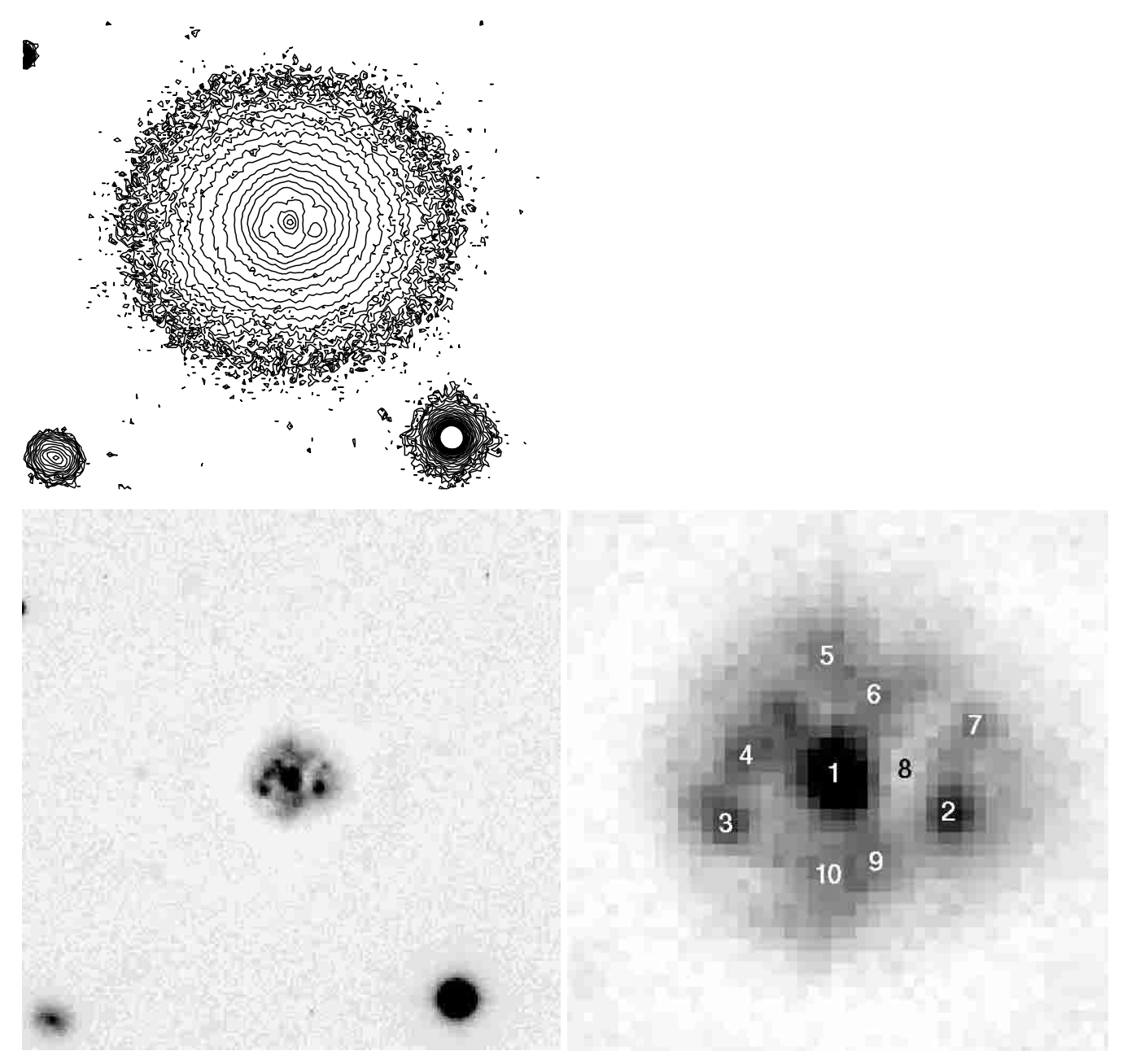

Fig. 2. Top: $1^{\prime} \times 1^{\prime}$ contour plot $\left(g\right.$ band) of N50. The faintest and brightest contours displayed are 25 and 20 mag arcsec ${ }^{-1}$, respectively, with $0.25 \mathrm{mag} \operatorname{arcsec}^{-1}$ separation between concentric contour levels. North is up and East to the left. Bottom left: greyscale plot after subtraction of a Sérsic model. Orientation and scale are the same as in the upper panel. Bottom right: enlarged view $\left(15^{\prime \prime} \times 15^{\prime \prime}\right)$ of the central region with identification for the knots (see Table 1)

The bottom panel shows the $g-i$ colour profile (after slightly degrading the $i$ frame to the $g$ PSF to consistently sample surface luminosity). Note in the figure the blue colour bump about $3^{\prime \prime}$ from the centre due to the knotty ring. A smooth colour gradient is also evident along galaxy radius with the outermost regions sensibly bluer than the centre. This colour gradient affects the value of the parameter $N$, which increases monotonically from $N=1.39$ in $g$ to $N=1.63$ in $z$, sampling the range $8^{\prime \prime} \leq r \leq 28^{\prime \prime}$.

The location of N50 in a $g-r$ vs. $g-i$ colour diagram is shown in Fig. 4. In the main panel of the figure we compared galaxy integrated colours with the locus of Main Sequence stars (based on the Vilnius spectral atlas of Straizys \& Sviderskiene 1972), as well as with the theoretical colours for 15 Gyr template galaxies of different morphological type according to the three-zone synthesis models of Buzzoni $(1998,2000)$. As expected, N50 colours are slightly bluer than high-mass ellipticals, and intermediate between $E$ and $S a$ Hubble types.

A more detailed match of the population synthesis predictions with the N50 colour profile and with the nuclear knotty features is attempted in the insert panel of Fig. 4. Buzzoni's (1989) simple stellar population (SSP) models, computed for a Salpeter IMF, red horizontal branch morphology, and different metallicity $([\mathrm{Fe} / \mathrm{H}]=-0.25,0.0$, and +0.30$)$ are reported, tracking evolution from 5 to 15 Gyrs.

N50 aperture photometry at $2.5,10^{\prime \prime}$, and $30^{\prime \prime}$ is displayed together with individual photometry of the visible knots according to the identification number in Table 1 (see also the reference map in Fig. 2). A substantial agreement seems to exist between theoretical models and observations within the zero-point uncertainty in the magnitude scale. An old (10-15 Gyr) stellar population with slightly sub-solar metallicity $([\mathrm{Fe} / \mathrm{H}] \sim-0.2)$ appears to be the main component in $\mathrm{N} 50$ but a mild $[\mathrm{Fe} / \mathrm{H}]$ radial gradient might also exist inducing the blueing colour drift along galaxy radius.

Quite interestingly, nuclear knots reveal a much larger (and statistically significant) spread in colour. Knot \#1 (the nucleus?) appears indeed even redder than the galaxy core, as do those lying close to the apparent dust lane (\#8) 

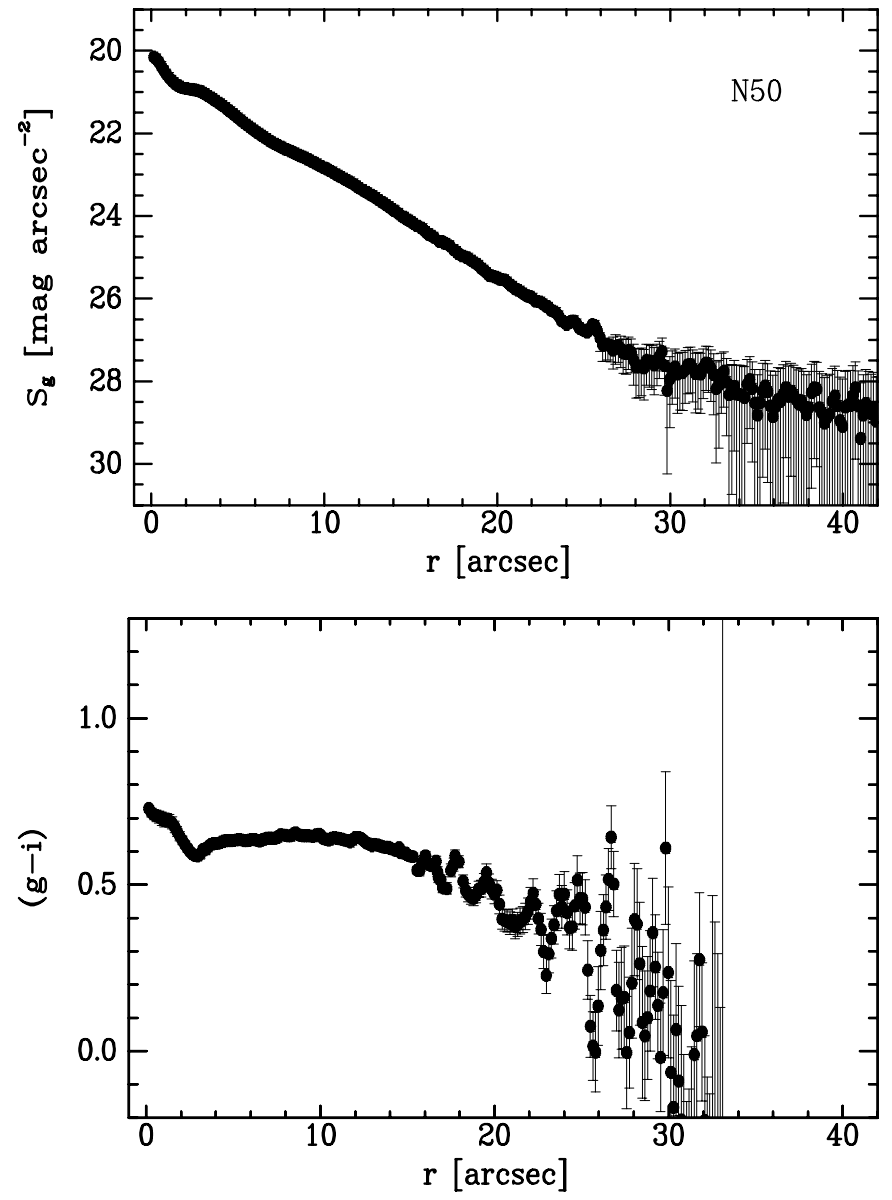

Fig. 3. Top: the $g$-band surface brightness profile of N50. Error bars account for photometric errors and uncertainties in the sky level. Bottom: the resulting $g-i$ colour profile of the galaxy

visible one arcsec west of the nucleus (cf. Fig. 2). Dust reddening might be "patchy" on the N50 central region, with the west area (corresponding to knots \# 1, 6, 7, near the dust lane \# 8) slightly more obscured $[\Delta E(B-V) \sim$ 0.05] than the east side (i.e., about knots \# 3 and 4 ).

Data in Fig. 4 are not corrected for our own Galaxy reddening (which however should not exceed $E(B-V) \sim$ 0.03 according to Burstein \& Heiles 1982). In addition, one should also consider a little blue shift of all the galaxy data by $\Delta(g-r) \simeq 0.012$ and $\Delta(g-i) \simeq 0.015$ to take into account for k-correction. Even correcting for these effects, it seems likely however that the whole stellar population in N50 should consist of stars older than 5 Gyr, and only a much enhanced (super-solar) metallicity should be invoked to predict a younger age.

The spread in age among the galaxy stellar population is even more evident from the analysis of the integrated spectrum, shown in Fig. 5. Galaxy spectral energy distribution has been obtained by matching the CASLEO data in the range $3500 \leq \lambda \leq 4400 \AA$ with ESO observations $(4400 \leq \lambda \leq 6300 \AA)$, adding then the monochromatic fluxes from the integrated $g, r, i$, and $z$ magnitudes. Gunn photometry also set the absolute flux calibration

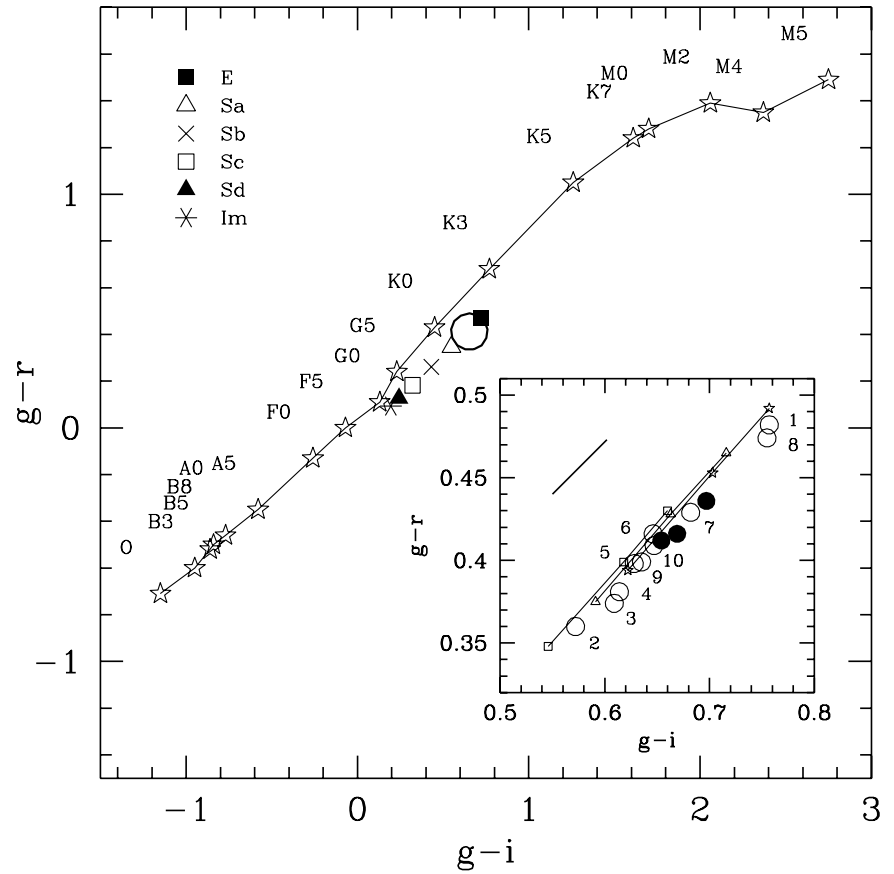

Fig. 4. (Main panel) - Two-colour diagram comparing N50 integrated $g-r$ and $g-i$ (big open dot) with the stellar Main Sequence from the Vilnius spectral atlas (star markers) and with Buzzoni's $(1998,2000) 15$ Gyr galaxy models for different Hubble types, as labelled top left. (Insert panel) - N50 aperture photometry (solid dots) and individual colours of the visible knots in the galaxy central region (open dots with identification labels according to Table 1). Aperture photometry is for circular spots at $2^{\prime \prime} .5,10^{\prime \prime}$, and $30^{\prime \prime}$ from galaxy centre. Colours become bluer at larger distance. Buzzoni's (1989) SSP evolutionary sequences for 5, 10, and 15 Gyr (in the sense of redder colours) are superposed (thin solid lines). Model metallicity is for $[\mathrm{Fe} / \mathrm{H}]=-0.25$ (squares), 0.0 (triangles) and +0.3 (stars). A reddening vector for $E(B-V)=0.03$ mag is displayed top left in the panel

reproducing galaxy energy distribution within the central $15^{\prime \prime}$ aperture.

Two SSP models from the Buzzoni (1989) data set, with $[\mathrm{Fe} / \mathrm{H}]=-0.25$ and age 5 and 15 Gyrs, are superposed on the plot. The older model fits well along the optical and red wavelength while it lacks UV luminosity below $4500 \AA$. On the contrary, the 5 Gyr stellar population provides a good fit to the ultraviolet but it would predict too blue Gunn colours. Accounting for SSP luminosity evolution, in a simple interpretative scheme assuming a mix of these two main stellar components, this allows us to estimate that the old $(15 \mathrm{Gyr})$ population comprises about $3 / 4$ of the total mass of the galaxy.

The redshift of $\mathrm{N} 50$, as derived from our spectra, amounts to $c z=2391 \pm 97 \mathrm{~km} \mathrm{~s}^{-1}$ (that is $z=$ $0.0080 \pm 0.0003)$. This yields a distance to the galaxy of $23.9 h_{0}^{-1} \mathrm{Mpc}$ and a distance modulus $(m-M)=$ $31.9-5 \log \left(h_{0}\right)$. From the model fit of Fig. 5, a bolometric correction to $g$ of $(\mathrm{Bol}-g)=-1.01$ can be obtained, leading for N50 to $M_{\mathrm{bol}}=-17.7+5 \log \left(h_{0}\right)$. This is $L_{\mathrm{bol}}=9.310^{8} h_{0}^{-2} L_{\odot}$ or $M_{B}=-16.1+5 \log \left(h_{0}\right)$. 


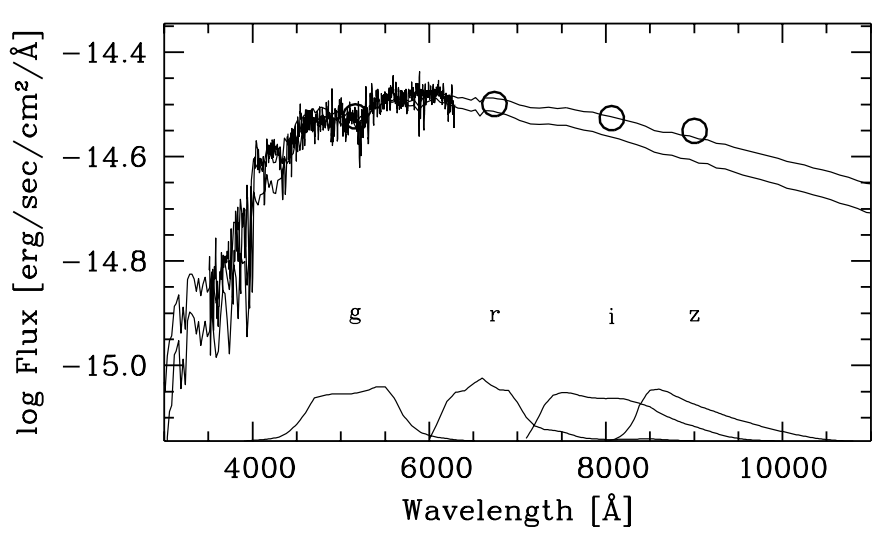

Fig. 5. Composite spectral energy distribution of galaxy N50. The CASLEO spectrum, in the range $3500 \leq \lambda \leq 4400 \AA$ has been matched to ESO observations $(4400 \leq \lambda \leq 6300 \AA)$ while integrated $g, r, i$, and $z$ Gunn magnitudes have been converted into monochromatic fluxes. Absolute flux calibration reproduces galaxy energy distribution within the central 15." aperture. Two SSP models from Buzzoni (1989), with $[\mathrm{Fe} / \mathrm{H}]=$ -0.25 and age 5 and 15 Gyrs, are superposed on the plot (thin solid lines, the older model is the "redder" one)

Estimating a theoretical $M / L_{\mathrm{bol}} \sim 5 \pm 1$ from the relevant SSP models of Buzzoni (1989) (once accounting for the whole stellar mass by integrating the Salpeter IMF) then one obtains $M_{\text {tot }} \sim 4.5( \pm 1.0) 10^{9} h_{0}^{-2} M_{\odot}$ as a fair estimate of the total (stellar) mass of N50.

\subsection{Star formation and morphology transition}

In its overall morphology, N50 is very reminiscent of the dwarf $\left(M_{B}=-16.71 \mathrm{mag}\right)$ galaxy Markarian 996, which Thuan et al. (1996) report to have smooth elliptical isophotes, with several bright knots and dust patches in its central $(\sim 400 \mathrm{pc})$ region. However, contrary to what we observe in N50, Mrk 996 clearly shows signs of active ongoing star formation in its centre, thus fitting with a nE BCD (nuclear-elliptical blue-compact-dwarf) classification.

The age we derive for the blue knots in N50 (see Fig. 6) suggests that it may have looked very similar to Mrk 996 a few Gyrs ago. Although now observed in its more quiescent evolutionary stage, the $\mathrm{H} \beta$ and $3727 \AA$ [OII] emission lines, prominent in our spectrum of the galaxy, still witness the presence of a wealth residual gas. In this sense, N50 probably represents an ideal link in the $\mathrm{dE}-\mathrm{BCD}$ connection (Thuan 1985; Evans et al. 1990; Meurer et al. 1992).

Quite importantly, it is also worth noting from Fig. 6 that even any massive intervening starburst activity older than 2 Gyr, superposed to the galaxy "quiescent" old stellar component, would still maintain integrated colours redder than $(B-V)=0.7$. According to the YC00 colour selection criterium, active dwarf ellipticals would therefore be recognized as "standard" galaxies in the $L-N$ relationship.

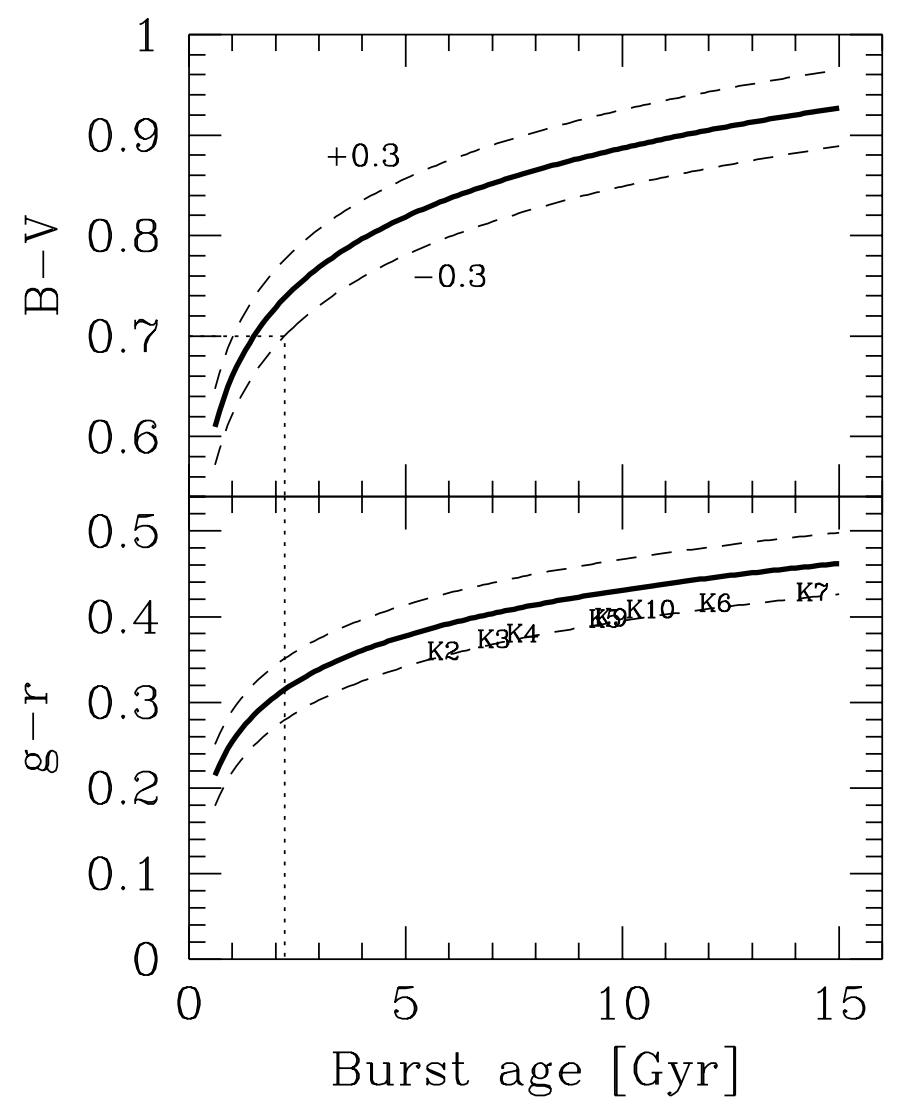

Fig. 6. Expected $B-V$ and $g-r$ star-burst colour evolution according to Buzzoni $(1989,1998)$. Three different values for $[\mathrm{Fe} / \mathrm{H}]=-0.3,0.0$ (solid lines), and +0.3 are displayed, as labelled on the $B-V$ plot. The indicative age distribution of the N50 central knots is also reported in the $g-r$ plot (assuming $[\mathrm{Fe} / \mathrm{H}]=-0.25)$. Note that, in general, even any massive starburst episode older than 2 Gyr, superposed to a galaxy old stellar component would not be able to turn integrated colours bluer than $(B-V)=0.7$ still allowing galaxy to be recognized as a quiescent "standard" elliptical according to the YC00 colour selection criterium

Despite such a negligible effect on the integrated colours, starburst episodes could however much more strongly affect galaxy surface brightness profiles. In the case of N50, for example, the presence of the bright knotty ring around the centre certainly modulates the Sérsic shape parameter leading to a higher fitting value for $N$.

\section{The $L-N$ relationship}

Each of the three "outliers" that depart from the $L-N$ relation in the NGC 5044 sample studied by C99 (namely N42, N49, and N50), displays a different kind of peculiarity. As shown in Sect. 2, N42 has a normal colour $(B-V=0.75)$, but its morphology reveals the presence of a central bulge that should be accounted for in a multicomponent fitting model.

On the other hand, N49 is a very blue $(B-V=0.49)$ irregular galaxy (C99), and cannot therefore be included in our analysis of the dwarf elliptical population. 
N50 takes only apparently the look of a standard dE object. In the previous section we showed that in spite of its quite normal $B-V$, this galaxy shows the signature of relatively fresh star formation in its centre.

Given a so wide range of morphological features it is difficult to firmly assess their systematic influence on the dE $L-N$ relationship. We should certainly agree with YC00 that any simple colour argument such as that relying on the integrated $B-V$, is not sufficient alone to secure a fair sample selection.

Attempting a very summary analysis in this sense, one could expect bulge-enhanced systems to affect the $L-N$ relation in a systematic way, especially at the bright tail $\left(M_{V}<-15\right)$ of $\mathrm{dE}$ luminosity function, by forcing a lower value of $N$ (see Fig. 3 in C99). While in some cases this could even (artificially) improve the match with the standard $L-N$ relationship, it does not help much for calibrating galaxy distance since in any case the Sérsic shape parameter poorly tracks galaxy luminosity at brighter magnitudes.

On the other side, intervening star formation, especially in case of clumpy features like those in N50, could dramatically affect galaxy surface brightness enhancing the spread in the profile fitting procedure. Again, this problem would more severly affect brighter dEs for which both "convex" and "concave" Sérsic profiles could result.

A fair estimate of the frequency of peculiar objects like N42 or N50 is in this regard the real concern to ultimately assess the reliability of the $L-N$ relation as a distance indicator. M32-like galaxies seem rather rare objects (Ferguson \& Binggeli 1994; Ziegler \& Bender 1998) but, as a matter of fact, even a rather coarse sample like that of C99 resulted affected by over $20 \%$ of such "deviating" objects.

In any case, it is clear from our results that any useful application of the $L-N$ relation as a distance indicator should forcedly be pursued on a statistical basis mainly relying on non-nucleated (faint) dEs. Obviously, such a tight sampling constraint might be the most stricking drawback of this approach for extragalactic studies.

\subsection{The $\alpha$ vs. $N$ coupling}

It is well known that reliable extragalactic distance indicators are either based on the luminosity of a given standard candle (e.g., SN Ia, globular clusters luminosity function, etc.), or on the relation between two independently measured parameters of galaxies, one depending on distance and the other distance-independent (e.g., the Tully-Fisher and $D_{n}-\sigma$ relations) (see for example Jacoby et al. 1992 or Trimble 1997 and references therein). For the $L-N$ relation to fulfill the condition of independence between both parameters, the total apparent magnitude should be obtained independently from $N$ by means of aperture or growth-curve photometry, instead of calculating it from the integration of Eq. (1), i.e.,

$V_{\mathrm{T}}=S_{0}-2.5 \log \left(2 \pi \alpha^{2}\right)-2.5 \log \left[\frac{\Gamma\left(\frac{2}{N}\right)}{N}\right]$.

However, as long as there is a good correspondence between total magnitudes obtained from Eq. (3) and from aperture or growth curve photometry (excluding nuclei, when necessary), integrated magnitudes should also work. Although the physical ground behind the $L-N$ relation is far from being understood, certainly Young \& Currie $(1994,1995)$ have made some interesting hypotheses in that direction, and first attempts towards a self-consistent theoretical scenario are due to Lima Neto et al. (1999).

The situation with the $\alpha-N$ relation is substantially different, since it involves two free parameters obtained from the same fitting formula. Worse, it is evident from Eq. (1) that the coupling between $\alpha$ and $N$ must be strong, i.e., any error in the measurement of $N$ will propagate (non linearly) to $\alpha$. This is important because there is a sizable scatter among values of $N$ measured for the same galaxies by different researchers (Ryden et al. 1999).

As an illustrative example, we have re-fitted from the C99 data the two high- $S / N$ profiles of dwarfs N29 $(N=0.54)$ and $\mathrm{N} 83(N=0.93)$ after varying the adopted sky level by a $\pm 20 \%$ of $\sigma_{\mathrm{SKY}}$ (this is a relative fluctuation $\Delta$ sky/sky $\sim 0.1 \%$ ). In addition, based on the C99 observational setup, we also generated an artificial profile for a faint $N>1$ galaxy and explored sky-fluctuation uncertainty likewise.

The results are displayed in Fig. 7 where solid bars represent the ranges spanned by the fitted Sérsic parameters. It is evident that variations in the adopted sky level cause the galaxies to move along the $\alpha-N$ relation (the dotted line is the polynomial fit for Fornax Cluster dwarfs given by $\mathrm{YC} 00$ ), showing that observational errors at least partially contribute to the $\alpha-N$ relation. This feature is to some extent a straightforward geometrical consequence of the fit: a more concave profile will generally predict a more "spiked" nucleus, that is a sharper pseudo scalelength.

Also shown in Fig. 7 (dashed lines) are the loci for galaxies with constant luminosity and $V_{\mathrm{T}} \propto S_{0}$, according to BJ98. The three dashed lines correspond to $V_{\mathrm{T}}=14$, 16 , and $18 \mathrm{mag}$, respectively, from upper-left to lowerright. Even before Sérsic law was first used to quantify the shape of $\mathrm{dE}$ profiles, it was qualitatively known that brighter dwarfs tend to be larger (and, at the same time, of higher surface brightness, and with more "concave" profiles; e.g., Ferguson \& Binggeli 1994, and references therein). Figure 7 shows, instead, that brighter galaxies following the $\alpha-N$ relation tend to have smaller $\alpha$ (and $N$ ) values (this can also be seen from the data in YC95; see also Jerjen \& Binggeli 1997). This is actually why the parameter $\alpha$ in the Sérsic law (Eq. (1)) can no longer be taken as a physical scalelength. Our suggestion is therefore that special caution should be deserved in using the $\alpha-N$ relationship to infer galaxies distances. 


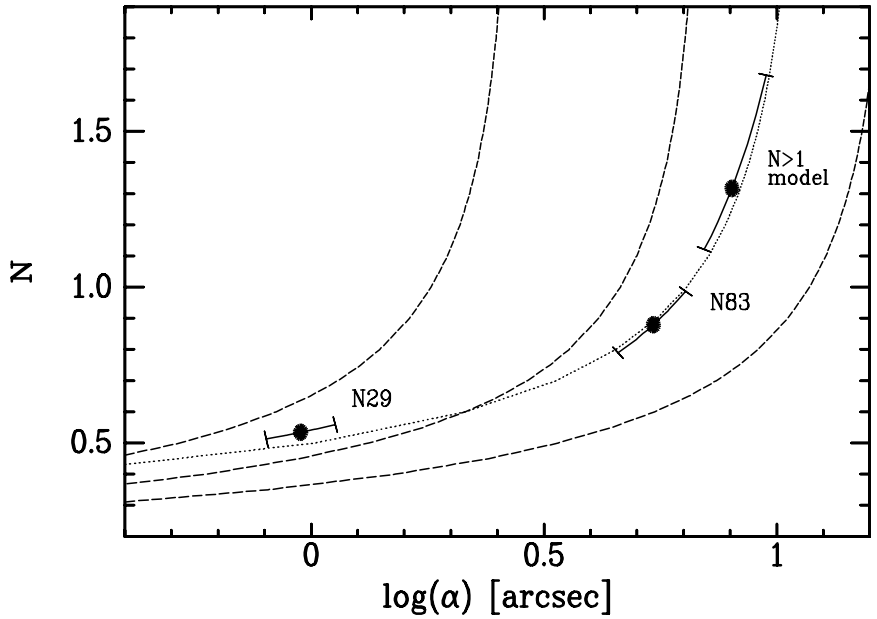

Fig. 7. Shape parameter $(N)$ vs. pseudo scalelength $(\alpha)$ for two galaxies in the C99 NGC 5044 Group sample and a simulated $N>1$ model. Bars represent the spanned range of the $(N, \alpha)$ parameters after varying the sky level by $\pm 20 \%$ of the sky rms. The dotted line is the YC00 polynomial fit to Fornax Cluster dwarfs. Dashed lines are galaxy loci for different constant luminosity (see text for details)

\section{References}

Andredakis, Y. C., Peletier, R. F., \& Balcells, M. 1995, MNRAS, 275, 874

Binggeli, B., \& Jerjen, H. 1998, A\&A, 333, 17 (BJ98)

Burstein, D., \& Heiles, C. 1982, AJ, 87, 1165

Buzzoni, A. 1989, ApJS, 71, 817

Buzzoni, A. 1998, in Cosmological Parameters and the Evolution of the Universe, ed. K. Sato (Kluwer, Dordrecht), 134

Buzzoni, A. 2000, A\&A, submitted

Buzzoni, A., Cellone, S. A., Forte, J. C., \& Saracco, P. 2001, in preparation

Caldwell, N., \& Bothun, G. D. 1987, AJ, 94, 1126

Caon, N., Capaccioli, M., \& D'Onofrio, M. 1993, MNRAS, 265, 1013
Cellone, S. A. 1999, A\&A, 345, 403 (C99)

Cellone, S. A., Forte, J. C., \& Geisler, D. 1994, ApJS, 93, 397

D'Onofrio, M., Capaccioli, M., \& Caon, N. 1994, MNRAS, 271,523

Durrell, P. R. 1997, AJ, 113, 531

Durrell, P. R., McLaughlin, D. E., Harris, W. E., \& Hanes, D. A. 1996, ApJ, 463, 543

Evans, Rh., Davies, J. I., \& Phillipps, S. 1990, MNRAS, 245, 164

Ferguson, H. C., \& Binggeli, B. 1994, A\&AR, 6, 67

Ferguson, H. C., \& Sandage, A. 1990, AJ, 100, 1

Jacoby, G. H., Branch, D., Ciardullo, R., et al. 1992, PASP, 104,599

Jerjen, H., \& Binggeli, B. 1997, Are "dwarf" ellipticals genuine ellipticals?, in Proc. of the Second Stromlo Symp., ed. M. Arnaboldi, G. S. Da Costa, \& P. Saha, ASP Conf. Ser., 116,239

Lima Neto, G. B., Gerbal, D., \& Márquez, I. 1999, MNRAS, 309, 481

Meurer, G. R., Freeman, K. C., Dopita, M. A., \& Cacciari, C. 1992, AJ, 103, 60

Papaderos, P., Loose, H.-H., Thuan, T. X., \& Fricke, K. J. 1996, A\&AS, 120, 207

Ryden, B. S., Terndrup, D. M., Pogge, R. W., \& Lauer, T. R. 1999, ApJ, 517, 650

Schneider, D. P., Gunn, J. E., \& Hoessel, J. G. 1983, ApJ, 264, 337

Sérsic, J. L. 1968, Atlas de Galaxias Australes, Observatorio Astronómico de Córdoba

Straizys, V., \& Sviderskiene, Z. 1972, Bull. Vilnius Astron. Obs., 35,1

Thuan, T. X. 1985, ApJ, 299, 881

Thuan, T. X., Izotov, Y. I., \& Lipovetsky, V. A. 1996, ApJ, 463,120

Trimble, V. 1997, Space Sci. Rev., 79, 793

Young, C. K., \& Currie, M. J. 1994, MNRAS, 268, L11 (YC94)

Young, C. K., \& Currie, M. J. 1995, MNRAS, 273, 1141 (YC95)

Young, C. K., \& Currie, M. J. 1998, A\&A, 333, 795

Young, C. K., \& Currie, M. J. 2001, A\&A, 369, 736 (YC00)

Ziegler, B. L., \& Bender, R. 1998, A\&A, 330, 819 